\title{
How, why, and when was the edgewise appliance born?
}

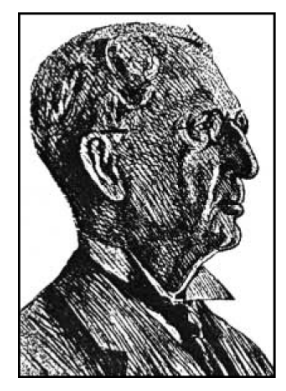

\author{
Julien PHILIPPE
}

\section{ABSTRACT}

Angle corrected anterior crowding by advancing the crowns of the incisors. For a long time he hoped the apices would follow along spontaneously. Then because of the uncertainty of this reaction, he started his quest for an appliance that would be able to accomplish root movement. The first one was difficult for orthodontists to manipulate, the second did not incorporate vertical control, but the third had all the qualities Angle was looking for. He died satisfied.

\section{KEYWORDS}

Bracket

Movement of the apex

Edgewise

Orthodontic mechanics

Ribbon-Arch. 


\section{1 - INTRODUCTION}

Angle created the edgewise appliance 80 years ago. It wasn't the fruit of a sudden inspiration. For years he had been searching for an appliance that would offer all the qualities he thought were needed in orthodontics. Many times he thought he had found it and he proposed systems that demonstrated improvement over their predecessors but finally turned out to be imperfect.

At the beginning of the twentieth century, Angle, an extremely idealistic man, counted on the aid of Mother
Nature $^{*}$ and believed that the Creator* shared his notion of an ideal treatment result. But, little by little, he realized he would have to correct the deficiencies of natural evolution with mechanics that were more and more sophisticated.

In order to understand how the edgewise appliance was born we must begin with the problems that Angle encountered when he utilized his expansion arch, the "E Arch." These problems illuminated the path that he followed until he reached the edgewise goal.

\section{2 - THE TREATMENT OF DENTAL CROWDING}

In 1907, Edward H. Angle published his most important work, the7th edition of his book1 that described his theory, his methods, and his technique, which was based on the "E- Arch" (fig. 1) and the use of inter-maxillary elastics.

To treat Class I cases that were frequently complicated by crowding without ordering extractions, which, by then, he had decided were inadmissible, the expanded the dental arches with the "E-Arch." The goal was to place all the teeth on the ideal curve of the arch that Angle called "the line of occlusion." Most of the time in order to arrive at this state, treatment had to include moderate transverse expansion and sometimes a considerable amount of labial movement of the incisors. Angle's book shows many treated Class I cases treated in this way with the incisor

(*) Angle always wrote "Nature" and "Creator" with an initial capital letter. teeth in pushed forward. Angle recognized this defect but he thought that, during the retention period, the anterior segments of the maxillae that had been compressed by the cause of the deformation, usually muscular pressure, would assume their normal shape, the one Nature had planned for them, thanks to a compensatory growth, which, in a few words, expresses Angle's fundamental belief. This new growth would permit the teeth that had been tipped forward by treatment to return to their normal inclination, as he put it, "The very apices of the roots, then so convergent, would ultimately be moved by Nature to their full normal upright positions" (Angle": p. 344).

Modern practitioners will freely confess that that type of spontaneous movement of root apices is far from the outcome they most frequently observe. Angle came to realize this, and without in any way changing his fundamental philosophy, 


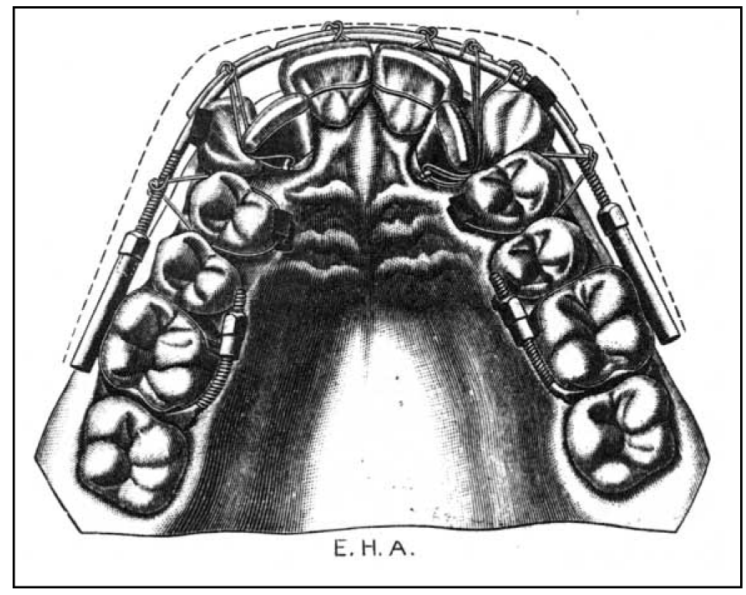

Figure 1

After Angle2. The "E-Arch." The arch can be advanced, and, therefore activated, in mouth by tightening screws with a key, without which it would be necessary to change the ligatures.

a new idea took possession of his soul, the dream of finding an "appliance to move the apices of roots." Numerous solutions popped into his fertile mind, but it is always a delicate matter for the head of a School to change his mind and abandon the system whose merits he had been preaching until then. Angle extricated himself from this trap skillfully, writing in 1912, "We know that working through the masticatory function, Nature will stimulate development and upright the roots of teeth until they return to their normal inclination,

\section{3 - THE "PIN AND TUBE APPLIANCE" (1912)}

Angle invented and introduced an appliance capable of fulfilling those objectives, the Pin and Tube appliance (fig. 2) in 1912. He called it a "bone growing appliance" because, he thought, in normal function its action of transmitting force to the roots would stimulate the growth of bone. In practice, the device turned out to be extremely difficult to manipulate. but this requires a great deal of time and demands a long and tedious retention period and, furthermore, success depends on growth of the bones, which is related to the age and the health of the patient." (Angle ${ }^{2}$ ). He continued, "Our treatment plan would be greatly improved if, instead of leaving the roots in an abnormal inclination to be uprighted by Nature during the retention period, those roots were moved bodily by a force so gentle and so well distributed that it would stimulate cellular activity and the growth of bone" (Angle², p. 855).
It was useful for an elite of technically group of especially dexterous practitioners; the others were unable to adjust it correctly and had many failures with its use.

Angle was, nevertheless, happy to have conceived of a new appliance rich with promise and to have caught up with the advances that his 

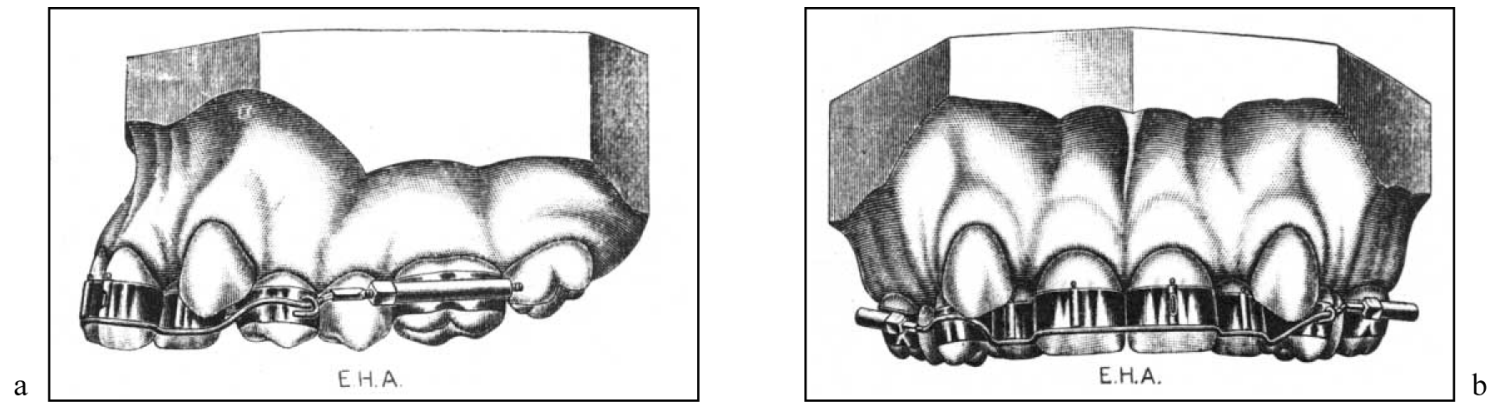

Figure $2 a$ and $b$

After Angle ${ }^{2}$. Activation of the screws is not enough. The stops must constantly be soldered in new positions or orientations.
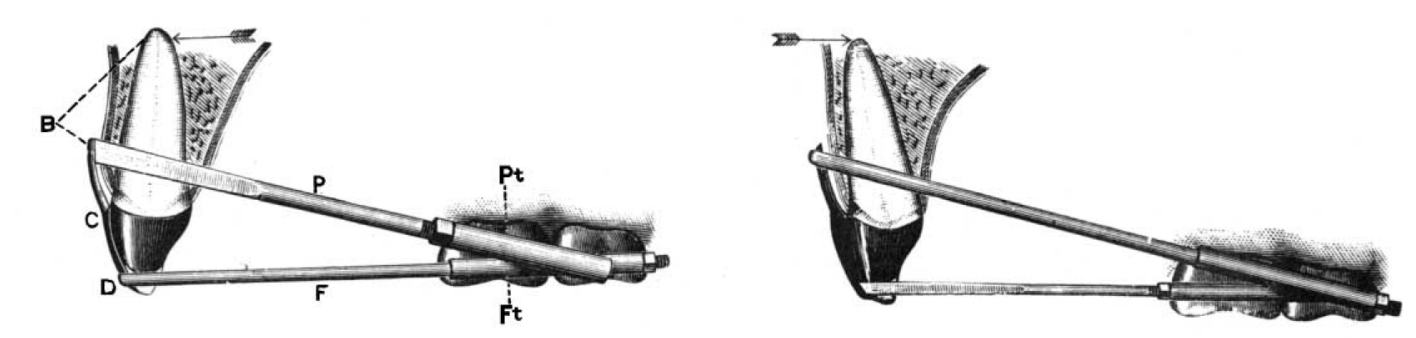

Figure 3

After C. Case. Calvin Case introduced a new system in 1893 that made it possible to move the apices of teeth by adjusting two "contouring" arches.

arch-rival Calvin Case had made when, in 1893, he proposed the "contouring" appliance (fig. 3), that was capable of moving the incisor roots. Today it is hard to imagine the frequency and the extent of movement that was ascribed to that appliance at the time. C. P Tacail, for example, in

\section{THE "RIBBON-ARCH"}

In 1916, Angle ${ }^{3}$ realizing how hard it was to regulate the Pin and Tube appliance, introduced a new appliance, the Ribbon Arch (fig. 4), with more of its components prepared by the manufacturer and far easier to handle than its predecessor. We describe its principle innovation, the
$1927^{14}$, showed a case of "upper retrusion" in which he had advance the crowns of the maxillary incisors $13 \mathrm{~mm}$ and their apices $10 \mathrm{~mm}$ (!). Even if these measurements are only approximate, they show what heroic objectives were aimed at.

bracket in an earlier article (Philippe ${ }^{13}$ ). This bracket was open occlusally and the ribbon arch was inserted lying flat. Only the eight incisors and the mandibular canines carried brackets. The bicuspids were not bracketed because it would have been impossible to insert the arch simultaneously 


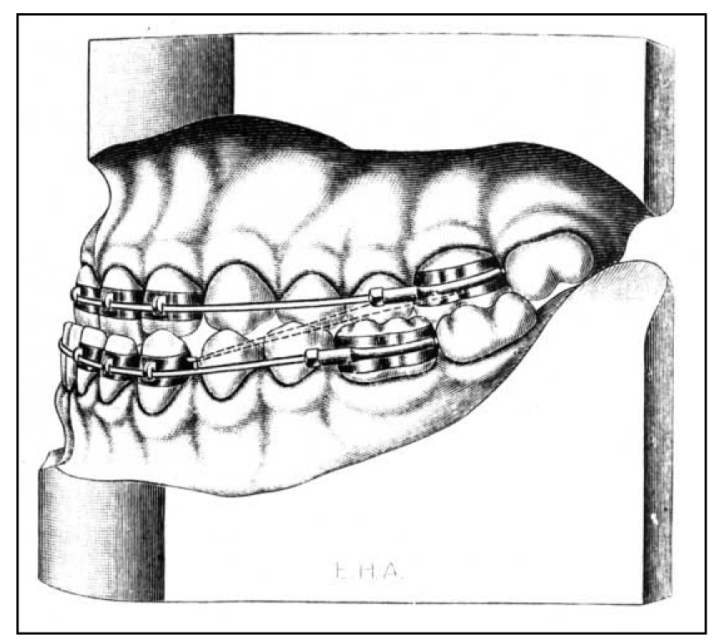

Figure 4

After Angle ${ }^{2}$. The Ribbon-Arch used in a treatment of a Class III case.

into the horizontal molar tube and into the vertical bracket of the adjacent pre- molar $\left(\right.$ Brodie $\left.^{9}\right)$. Owing to this conceptual deficiency, with no bearing on the bicuspid teeth practitioners found it very difficult to correct an excessive curve of Spee Brodie $^{8}$, Dewell $\left.{ }^{10}\right)$. But in other respects, its supple character making it effective in rotating incisors and for moving the roots of anterior teeth, it proved highly satisfactory to the practitioners who used it.

Accordingly, Angle sought a way to remedy this deficiency. He realized that he would be able to place the arch in brackets fixed to the bicuspids if they had another conformation, if, for example, their slots were horizontal as was the molar tube. Angle experimented with many models of premolar brackets and one of his students, Raymond Begg, complained of having to make them by hand!

It's possible that Angle might have conside- red that instead of trying to design a horizontal premolar bracket he could have placed a tube on the

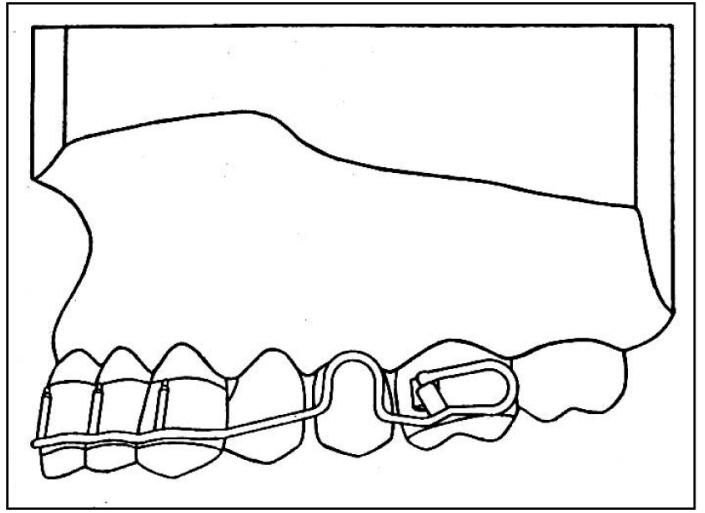

Figure 5

After Angle ${ }^{3}$. Proposition, rejected by Angle, of a Pin-and-Tube system with a vertical molar tube. The arch would have been adjusted in an anteroposterior direction by bends made in the vertical loop and not by screws.

molars that was vertical like the brackets. But Angle 6 had already thought about and rejected this solution in the Pin and Tube appliance (fig. 5). He was afraid that the vertical end of the arch, bent into the indispensable vertical loop and affected by the expansion incorporated in the arch, would be unable to retain the same orientation throughout treatment. This would provoke round trip, or back forth, movements that would be harmful to "the cells of the periodontal ligament and to the alveolar bone" causing a grave risk to the integrity of the molar anchorage.

Finally his experiments with premolar bracket design were crowned with success. And the new bracket seemed to be so eminently suitable that Angle thought about extending its use to all the teeth (evidently the mixture of horizontal and vertical brackets would not make the orthodontist's task an easier!) So at first, according to Dewel ${ }^{10}$. "Angle initially conceived of the edgewise 
bracket as a complement to the Ribbon Arch, not a substitute for it." But Angle soon realized that in addition to its ease of manipulation, the edgewise bracket offered many other advantages, in particular the capability of controlling mesio-distal inclination of teeth and vertical movements.

\section{5 - THE EDGEWISE APPLIANCE (1928)}

Even if the consideration we'll discuss in a moment was not the determining factor in the development of the edgewise appliance, it must be noted that Angle was a man who never neglected the commercial aspect of his profession. Among other signs of this character trait, remember that he patented each new orthodontic device he introduced, 32 in all, and they earned royalties for him (Matasa and Graber $^{12}$ ) The idea of having the components of an appliance made in a factory was, without doubt, an indication of progress for orthodontics but it was also a source of income for Angle. And we can see that as the E-Arch evolved into the Edgewise by way of the Pin and Tube and Ribbon arches, more and more components were being manufactured and not fabricated by hand in orthodontic offices.

The Edgewise appliance was developed progressively. Here's what J.S. Kloehn11 says about it, "In 1925 Angle began to develop the edgewise appliance. This labor was delegated to Alan Brodie. The appliance was made by hand and the brackets were cut out and shaped one by one. The conformation of the bracket was constantly modified and improved. In 1926, Angle decided that the appliance was ready, ready for clinical trials. And Brodie for the first time treated a patient with the "new mechanism" as it was described at the time. The annual convention of Angle students of 1928 took place on June 28. It began with a lecture by Angle on the new mechanism and ended with Brodie's presentation where he said "the arch wire we tested brought us something we had never known before, the complete control of all the teeth individually and collectively at the same time." Angle then wrote a long article with the help of Charles Tweed who had just finished his training in orthodontics. It was published in "Dental Cosmos" in December 19284, February ${ }^{5}$, March ${ }^{6}$, and April ${ }^{7}$ 1929. The Edgewise had been born officially.

It must be said the Kloehn's chronology, even though it is based on the best available eye witnesses, does not match well with the information Angle presented in his article. Angle said that he presented a first draft on the new mechanism in 1925, which would mean its conception was already well advanced by that date. At the end of the 1928-1929 article five cases are presented. Their dates are clearly visible on pictures of the models, beginning of treatment May 1924 end of treatment in November 1924 (!) In addition, the many details that Angle elucidated in his article would suggest a considerable experience in the manipulation of the appliance and reflect incidents occurring in its use in daily practice. The chronology that Kloehn presents seems a little behind and indicates 
that the gestation period for the appliance was quite short. But in a lecture he gave in 1929 Brodie said, "Dr. Angle began to work on this new appliance ten years ago."

If Angle was thinking about the appliance for years before he intro- duced it, he was not granted much time to defend it. He died on August 111930 saying, "I have finished my work and I did my best."

At that moment another long, a very long history began, the history of the Edgewise appliance.

\section{REFERENCES}

1. Angle EH. Treatment of malocclusion of the teeth. 7th éd. Philadelphia: S.S.White Dental Mfg Cy, 1907.

2. Angle EH. Evolution of orthodontia. Recent developments. Dental Cosmos 1912;54:853-67.

3. Angle EH. Some new Forms of Orthodontie Mechanism, and the Reasons of their Introduction. Dental Cosmos 1916;58:969-94.

4. Angle EH. The latest and best in Orthodontic Mechanism. Dental Cosmos 1928;70:1143-56.

5. Angle EH. The latest and best in Orthodontic Mechanism. Dental Cosmos 1929;71:164-74.

6. Angle $\mathrm{EH}$. The latest and best in Orthodontic Mechanism. Dental Cosmos 1929;72:260-70.

7. Angle $\mathrm{EH}$. The latest and best in Orthodontic Mechanism. Dental Cosmos 1929;73:409-21.

8. Brodie AG. A discussion on the Newest Angle Mechanism. Angle Orthod 1931;1:32-8.

9. Brodie AG Orthodontic Concepts Prior to the Death of Edward Angle. Angle Orthod 1956;26:144-55.

10. Dewell BF. The Ribbon-Arch, and its Influence in the development of Orthodontic Appliance. Angle Orthod 1981;51:263-8.

11. Kloehn JS. Portrait of a Giant. Angle Orthod 1990;60:129-35.

12. Matasa CG, Graber TM. Angle the innovator, mechanical genius and clinician. Am J Orthod Dentofac Orthop 2000;117:444-52.

13. Philippe J. Qui a inventé le bracket ? Rev Orthop Dento Faciale 2002;36:365-9.

14. Tacail CP. Correction d'un cas de rétrognathisme supérieur. Orthod Fr 1927:126-45. 